\title{
Dysphonia due to tremor
}

\author{
G. ARDRAN, M. KINSBOURNE, AND GEOFFREY RUSHWORTH \\ From the Nuffield Institute for Medical Research, University of Oxford, and the Department \\ of Neurology, Churchill Hospital, Oxford
}

An account is given here of investigations performed on a patient with familial tremor affecting the voice so severely as to make her speech almost unintelligible.

Interruption of speech is not uncommon in senile tremor and in Parkinsonian tremor, but this is not severe enough to give rise to difficulty in communication. In familial tremor (Larsson and Sjögren, 1960) the voice is affected in a minority of cases (Brown and Simonson, 1963) due, in Critchley's (1949) opinion, to tremor of the muscles of the larynx, lips, tongue, and diaphragm, separately or in combination. Although the characteristics of the interrupted speech have been studied by means of visual representation of tape-recorded speech (Brown and Simonson, 1963) the muscles responsible for tremor of speech do not appear to have been directly studied by modern methods.

Below are reported cineradiographic, electromyographic, and other studies of a particularly striking case of familial tremor involving the voice. The tremor was so severe as to produce what sounded like a rapidly recurrent sequence of glottal stops, interrupting speech so frequently as to make it difficult to understand.

A.A., aged 72, a housewife, was admitted under the care of Professor W. Ritchie Russell on account of a tremor of long standing, which, in the last six months, had become more generalized and severe. This began very gradually at about the age of 50 , affecting both hands as well as the face, and was more marked on the right side. It remained very slight until 10 years ago when, at about the time of her husband's death, it began to affect her voice, giving her increasing difficulty in pronouncing her words over the intervening period. Since then, in the last six months it had become considerably more obtrusive, and was also noticeably affecting her legs.

Except for lumbago, recurrent varicose veins, and bunions (for which she had hemiphalangectomy), she had, in all other respects, been in good health, without relevant incident in her past history. There was, however, a strong family history of tremor (Fig. 1). Her mother had a facial, voice, and severe limb tremor. Of her eight siblings, her eldest brother, now deceased, had a mainly right-sided tremor for many years. Of the patient's three

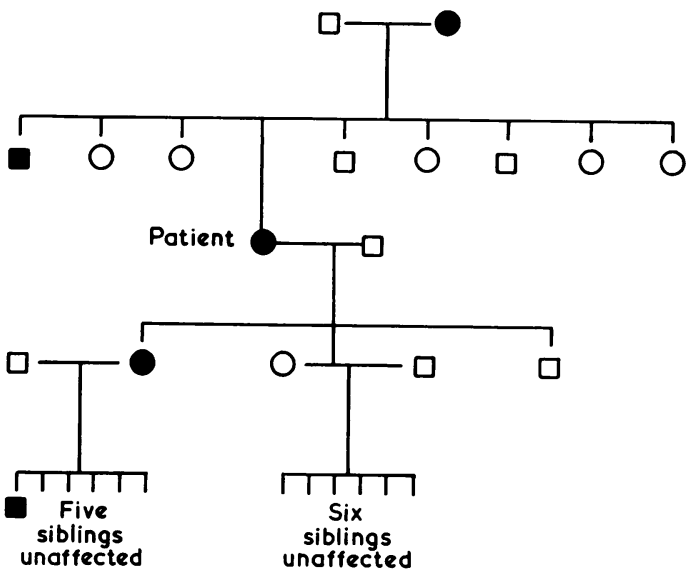

FIG. 1. Pedigree of the family. Those marked with black affected by tremor.

children, all of whom have been interviewed, the eldest, an unmarried man aged 51, has a limb tremor. Her elder daughter, now a married woman aged 48 , complains of a mild tremor affecting the face and limbs since the age or 30. The oldest of her six children, a boy, was first thought to be affected at the age of 15 . The presence of abnormal tremor was confirmed clinically and electromyographically in the first but not the other two cases. No history is available about the patient's grandparents or about her nephews or nieces.

On examination she was a thin, lively, intelligent and agreeable woman who did not look her age. Her blood pressure was $170 / 90 \mathrm{~mm}$. $\mathrm{Hg}$. There were no abnormalities on general examination. On neurological examination, the tremor was the only abnormality. A regular static tremor, increased in amplitude by movement, affected her arms and outstretched hands, causing rapidly alternating pronation and supination displacement at the wrists and an abduction adduction alteration of the fingers. The tremor was present, though less marked, in the legs, and was of greater amplitude in the right than in the left-sided limbs. The facial muscles and muscles of mastication, especially on the right, were subject to tremor, as was the tongue, and there was a regular titubation of the head. The hyoid bone, larynx, and trachea could be seen and felt to rise and fall rapidly in sympathy with the tremor 
elsewhere. Her speech was quavering and was constantly interrupted by a succession of pauses which sounded like glottal stops. This made it difficult for her speech to be understood, especially when she was excited. Her voice was tape recorded and the tremor of limbs, head, tongue, etc., was filmed.

On investigation, a blood count and urine analysis gave normal results, and the cerebrospinal fluid was under normal pressure (constitution protein $45 \mathrm{mg}$./100 ml., no cells, Lange and Wassermann reactions negative). Lumbar air encephalography (Dr. P. W. E. Sheldon) revealed considerable dilatation of the body of the right lateral ventricle, but no other abnormality. Chromosome analysis of her blood (Dr. H. A. Johnston) showed modal number 46, with an apparently normal female karyotype and no evidence of chromosome abnormality.

The following special investigations were carried out:1 Direct $16 \mathrm{~mm}$. silent colour cinematography of the patient at rest and while talking, showing the tremor of the jaw, facial muscles, etc., at a rhythm of about 5-6 per second.

$2 X$-ray cinematography at 50 frames per second on $35 \mathrm{~mm}$. film, of the lateral projection of the tongue, soft palate, mandible, and larynx, together with a simultaneous sound recording on tape while attempting to say 'Ee'.

3 A limited cineradiographic examination of swallowing. 4 Antero-posterior tomography of the larynx during breathing, phonation, and straining.

5 Electromyography of several pairs of muscle.

6 Tremor recordings.

7 Examination of the patient's son, elder daughter, and grandson.

When attempting to say 'Ee', cineradiographic frames showed that the tongue, larynx, and soft palate assumed the normal 'Ee' position. The tongue assumed the characteristic shape, the soft palate elevated to close off the nose, the larynx and hyoid were elevated a little, and the arytenoid masses moved forward and downward and the vocal cords were approximated: this also reduced the width of the laryngeal vestibule.

While saying ' $\mathrm{Ee}$ ' there was a small movement of the tongue roughly upward and forward and back again, at about 6 per second. The soft palate fell slightly from the elevated position at the same time as the tongue moved $\underset{\mathbb{D}}{Z}$

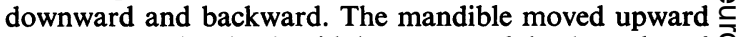
and backward. The hyoid bone moved backward and

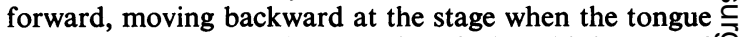
moved downward and backward, and when this happened the epiglottis was moved backward a little. This we believe to correspond with the break in phonation. The vocal folds, which turned downward and inward from the $\frac{3}{\mathrm{~g}}$. respiratory to the phonatory position, appeared to main- $\frac{\text { \% }}{7}$ tain the phonatory position throughout the act. This was confirmed by tomography which, although the dura- $\overrightarrow{\vec{F}}$ tion of exposure was of the order of one second, showed $\stackrel{5}{?}$ the vocal folds in the phonatory position. When the epiglottis is moved backward, this will reduce tension on the false vocal cords and reduce the size of the laryngeal $\frac{\bar{p}}{\vec{D}}$ vestibule. This is the mechanism for producing a partial $\stackrel{\varnothing}{\Omega}$ or complete glottal stop. It is very transitory. One would o expect that this would coincide with a reduction in ten- ${ }^{\infty}$ sion on the vocal cords associated with lack of activity of $\vec{O}$ the cricothyroid muscles.

A sound spectrograph was made of a tape recording of $\vec{\omega}$ the patient saying 'bait' (Fig. 2). Dr. A. J. Fourcin commented that, in his opinion, the wide-band spectrogram showed that, during the interruptions, air was still passing between the vocal cords and it is likely that these 0 were widely separated.

Cineradiographic examination of swallowing showe that the patient swallowed without any apparent diffi- $\overrightarrow{0}$ culty, although clearance from the mouth was not cong응 plete on the first swallow and might require a second one. This, however, may be due to her wearing dentures. Thec tremor is continuous throughout the swallowing ag, though it may diminish a little in amplitude from time time. There was no opening of the larynx during $\overrightarrow{5}$ swallowing.

ELECTROMYOGRAPHY This was performed by means of co-axial needle electrodes within the following pairs of
muscles:-

Left cricothyroid and right hyoglossus muscles In the⿳亠丷厂 hyoglossus muscle at rest, there was spontaneous motor $\stackrel{\square}{\complement}$ unit activity grouped into a tremor rhythm of 5-6 per $\overrightarrow{\vec{A}}$

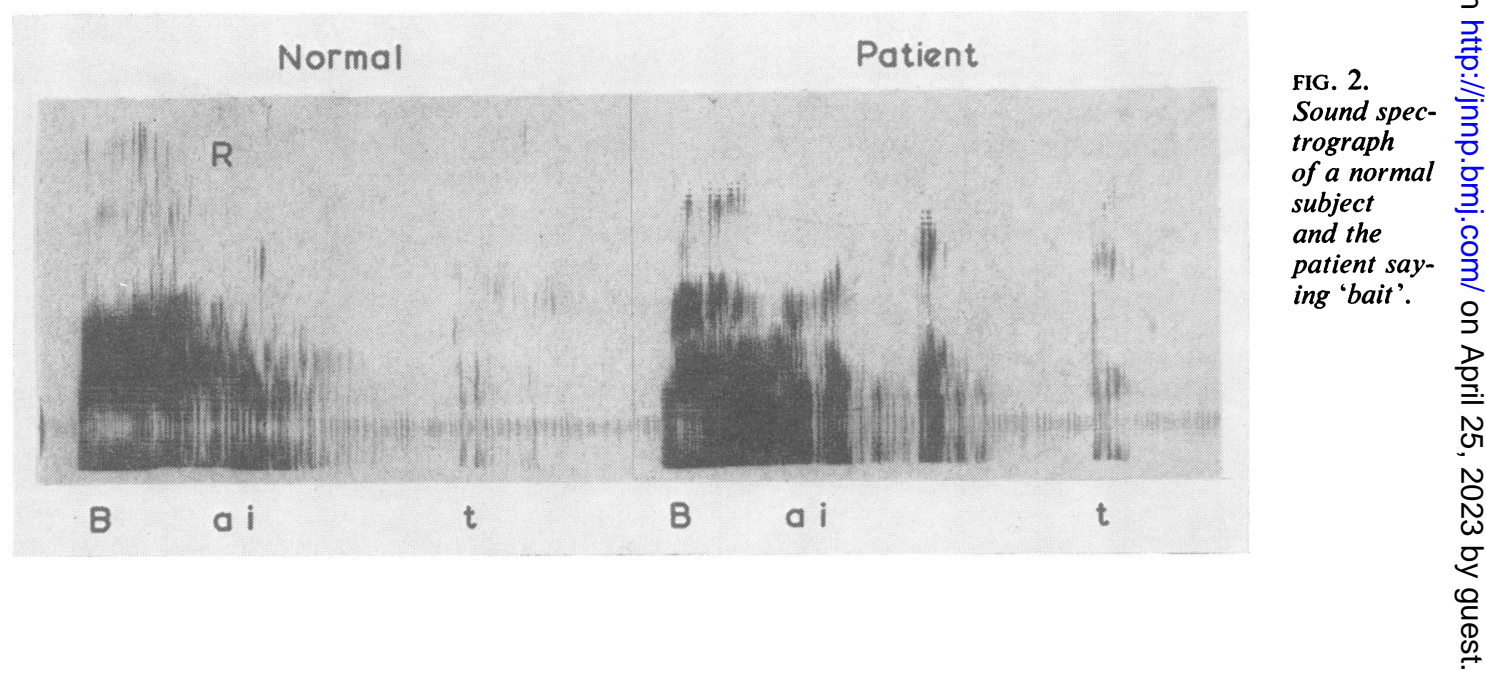




\section{Cricothyroid}

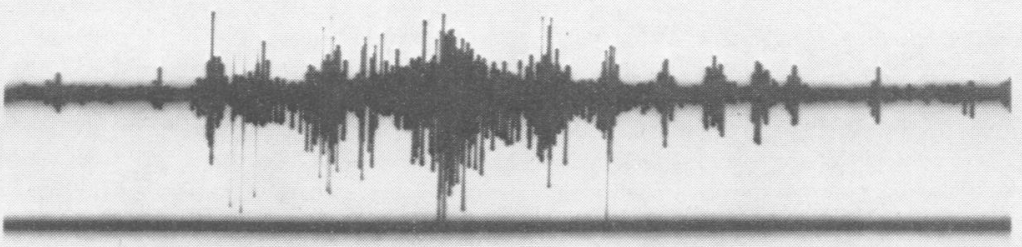

\section{I second}
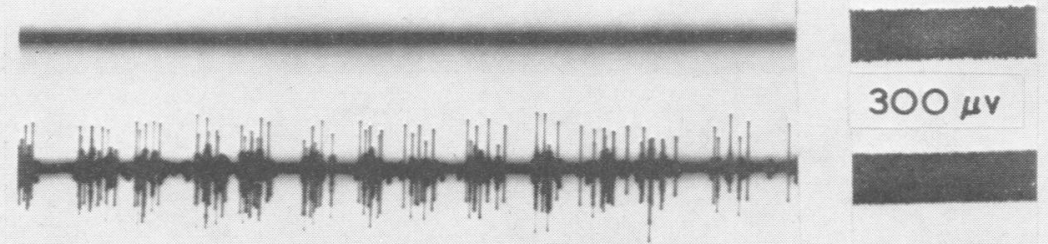

FIG. 3. Electromyograms recorded by means of coaxial needle electrodes. (a) Left cricothyroid. There is background activity grouped at about 6/sec., but an action tremor is obvious when the patient says 'Eee'. (b) Left hyoglossus at rest. There is a static tremor of about $6 / \mathrm{sec}$.

\section{L.Hyoglossus}

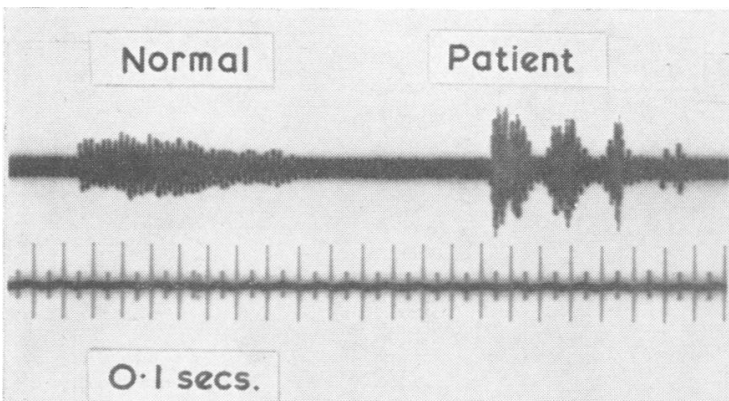

FIG. 4. Visualization of the patient's voice tremor. A normal subject, and then the patient, saying 'Eee' into a microphone, the output of which is displayed on a cathoderay oscilloscope. The tremulous response is obvious in the patient. second (Fig. 3). Each burst of activity was itself an asynchronous discharge of motor units which lasted 100-130 msec. The tremor rhythm was not precisely regular, and it occasionally disappeared entirely for several seconds at a time. It was not possible to identify the origin of this slow cyclical pattern. The tremor rhythm was briefly obliterated during swallowing.

In the cricothyroid muscle, spontaneous activity was only recorded occasionally and fewer motor units participated than in the hyoglossus, but they were grouped to form a tremor rhythm of about 5 to 6 per second (Fig. 3). The tremor in this muscle seldom occurred at the same time as that in the hyoglossus, but on one occasion, for a brief period only, static tremor was present in both muscles and it was then alternating in type.

When the patient attempted to say a long 'Eee', the cricothyroid response was extremely irregular, with the greatest discharge of motor units at the beginning and end of the effort. During the sound production the asynchronous discharges of motor units grouped themselves into a fairly regular tremor rhythm at a rate slightly faster $(8 / \mathrm{sec}$.) than the resting tremor. The individual bursts of 
action potentials occupied $60-100 \mathrm{msec}$. and involved many motor units. (When the sound made by the patient was picked up by a microphone and displayed on a cathode-ray oscilloscope, the same rate of sound interruption was found (Fig. 4)).

Right pectoralis major and right inspiratory intercostal muscles There was a $6 / \mathrm{sec}$. tremor in the pectoralis at rest, which disappeared on voluntary effort.

From the intercostal muscle a single unit discharged on inspiration at 13 per second on a very low amplitude background at $6 / \mathrm{sec}$., which continued throughout inspiration and expiration.

Right tibialis anterior and right gastrocnemius and left tibialis anterior and left gastrocnemius When the limbs were at rest and supported there was no spontaneous activity in any of these muscles. If, however, the limb was voluntarily lifted, then a tremulous response occurred io the tibialis with action potentials grouped into a rhythn of $6 / \mathrm{sec}$., superimposed on a background of irregulare low-amplitude motor unit discharges. With more intens activity, such as increasingly resisting the examiner, the same tremulous response occurred and, finally, the antagonistic muscles came into action with a simila tremor rhythm which alternated with that of the agonista.

On sudden passive dorsiflexion of the foot (Fig. 5a) Westphal's paradoxical contraction of the tibialis anterion: occurred. It was more marked on the left, and was main $\overrightarrow{\bar{\sigma}}$ tained for several seconds. During the maintained part of this reflex, the action potentials were grouped into tremor rhythm of $6 / \mathrm{sec}$. (Fig. 5b).

\section{R. Tibialis Anterior}

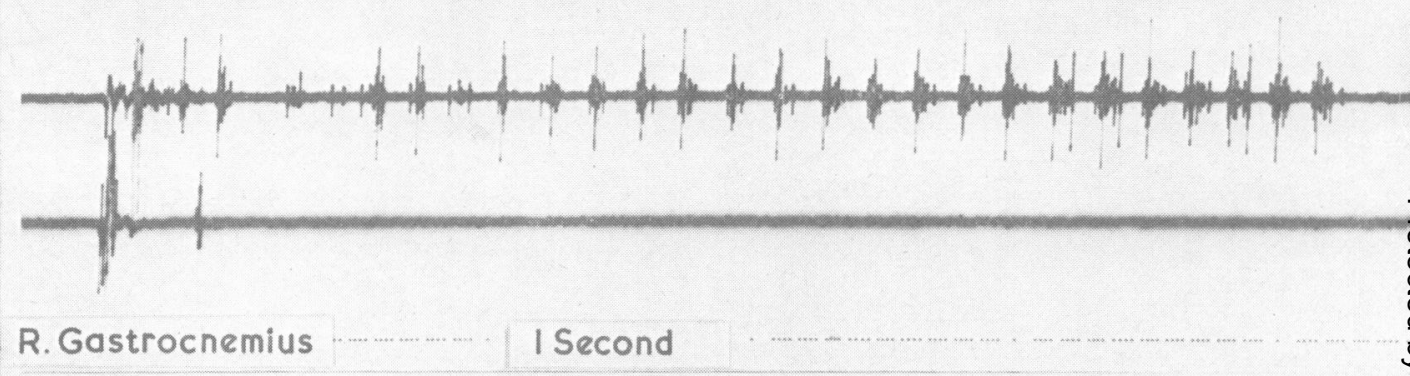

R. Gastrocnemius

I Second
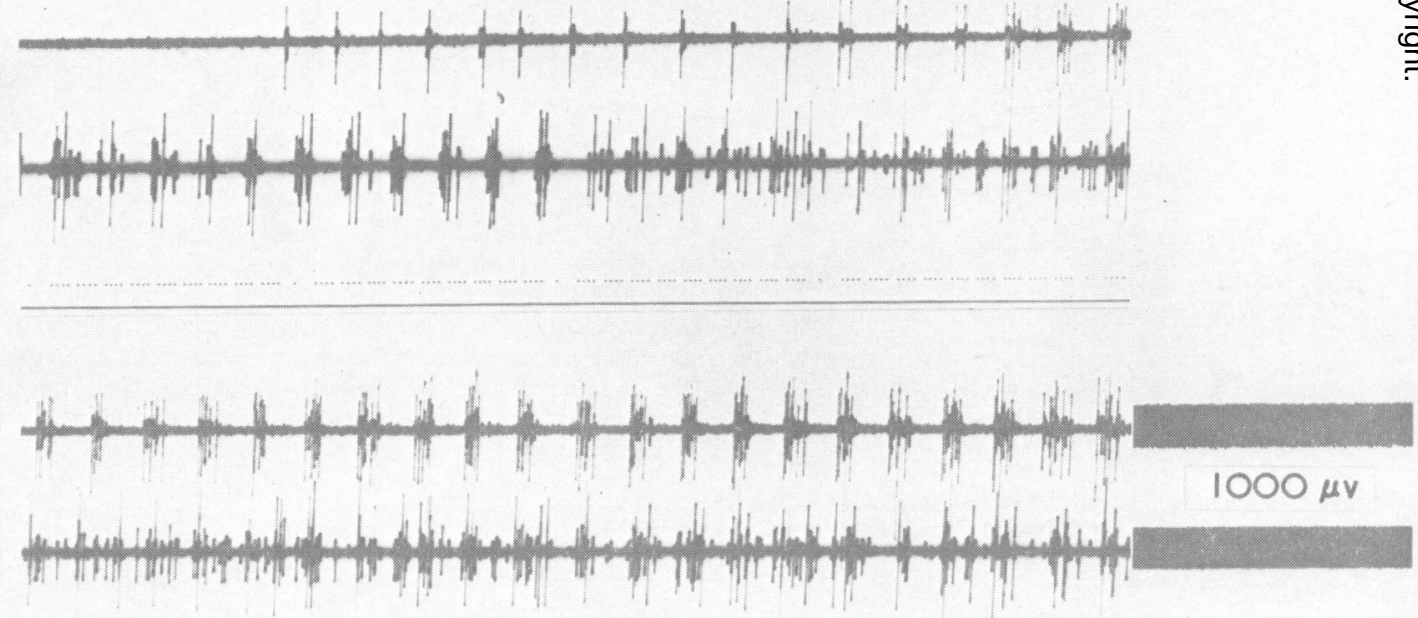

FIG. 5. Simultaneous electromyograms from right tibialis anterior and right gastrocnemius. (a) Sudden passive dorsit flexion of the foot produces a brief phasic stretch reflex in the gastrocnemius which just precedes a burst of activity in the tibialis anterior (Westphal phenomenon or paradoxical contraction) which is long, continuous, and tremulous. (b) and (c) Increasing contraction of the gastrocnemius. Initially only the gastrocnemius responds, and some action tremor i $\aleph^{-}$ apparent. With greater effort, the tibialis anterior also contracts with clear tremor grouping at 6/sec. 

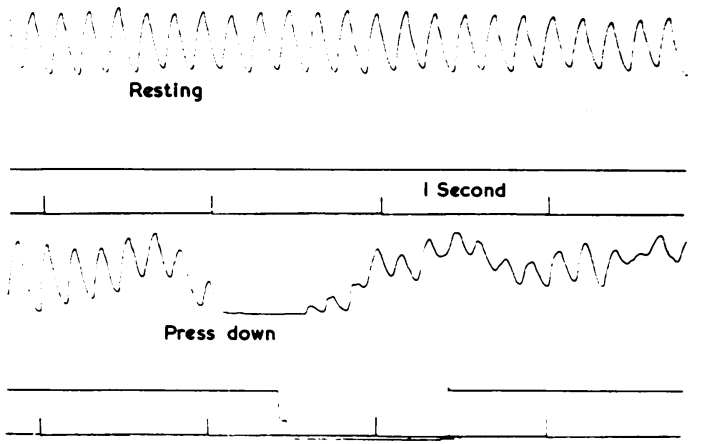

FIG. 6. Resting tremor recorded from the unsupported arm by means of a ceramic disc on which the patient's first finger rested. Extremely regular tremor, both in amplitude and rate (6/sec.). Muscular activity (pressing down with finger) increases the tremor rate slightly and its amplitude becomes irregular.

In the right arm the resting postural tremor of the shoulder muscles was recorded by asking the patient to place her right forefinger very gently on a ceramic disc transducer (Rushworth and Willison, 1960), the arm remaining unsupported. A tremor rhythm of 6 per sec. was recorded, which was extremely regular in both rate and amplitude (Fig. 6). When the patient pushed gently on the disc, the tremor amplitude became irregular, but the rate remained at 6 per second.

With the arm supported, the tremor recorded from the right first finger was not quite so regular in amplitude as before, but its rate was 6 per second, and this rhythm was also dominant when the patient attempted gentle flexion of the forefinger against the disc.

\section{EXAMINATION}

Recordings were also made from the following relatives:1 Son, L.W.H., aged 51. Recordings made from right and left forefinger. At high gain, there is a suspicion of a 6 per second rhythm at rest, which speeds up into the normal range on pressing down the disc.

2 Daughter, P.L.S., aged 46. No evidence of low-frequency tremor, even at high gain.

3 Grandson, L.S., aged 24 . No evidence of low-frequency tremor, even at high gain.

\section{DISCUSSION}

While voice tremor has been repeatedly reported, its severity in the individual cases is difficult to deduce from descriptions in the literature. It seems likely that familial tremor so severe as to make speech well-nigh incomprehensible is exceedingly rare.

A number of different muscle groups could conceivably rhythmically interrupt the air stream mediating voice in subjects with tremor. Clinical examination cannot distinguish between these possibilities. Cineradiography and electromyography permit a closer scrutiny of the action of individual muscle groups. In the event, multiple possible causes for voice interruption were found in our patient. The tongue movement, the fall of the soft palate allowing nasal escape of air, glottal stopping, the rhythmical reduction in tension of the vocal folds due to cricothyroid action - even, perhaps, the chest tremor, or a combination of these-could have contributed. Rhythmical pressure or tension on the floor of the mouth, the hyoid bone, the skin over the larynx, or thumping the chest, can all produce somewhat similar bleats or breaks in phonation. Such changes can also be produced during similar acts while the individual is whistling, which is not supposed to involve any laryngeal activity. However, the spectrographic findings showed one possible cause: complete apposition of the vocal cords did not occur, as the air stream was not completely blocked during the interruption in speech. On balance, the most likely cause of the interruptions of voice was, on the contrary, a rhythmically recurrent overwide separation of the vocal cords.

\section{SUMMARY}

A woman, aged 72 , was found to suffer from severe generalized familial tremor, notably affecting the voice so as to interrupt speech and making it difficult to understand. The relationship between voice tremor, tremor in vocal and respiratory muscles, and the patency of the air passages was examined by cineradiography, electromyography, sound spectrography, and other methods. The interruption of voice appeared to be due to rhythmic wide separation of the vocal cords.

We are grateful to Professor W. Ritchie Russell for permission to study and report this case; to Mr. D. Garner for performing the spectrographic analysis; to Dr. A. J. Fourcin for interpreting the spectrograms; and to Dr. H. A. Johnston for the chromosome analysis.

\section{REFERENCES}

Brown, J. R., and Simonson, J. (1963). Organic voice tremor. A tremor of phonation. Neurology (Minneap.), 13, 520-525.

Critchley, M. (1949). Observations on essential (heredo-familial) tremor. Brain, 72, 113-139.

Larsson, T., and Sjögren, T. (1960). Essential tremor. A clinical and genetic population study. Acta psychiat. neurol. scand., 36, suppl. 144.

Rushworth, G., and Willison, R. G. (1960). A method of recording small movements. Lancet, 1, 632-633. 